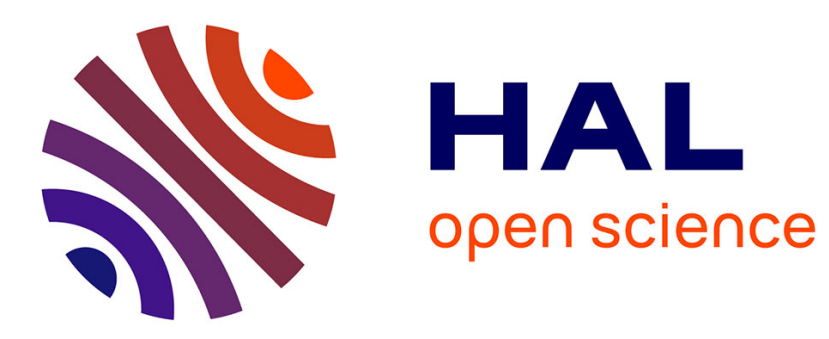

\title{
Model predictive real-time controller for a low-consumption electric vehicle
}

\author{
Tatiana Manrique-Espindola, Hugo Malaise, Mirko Fiacchini, Thomas
}

Chambrion, Gilles Millérioux

\section{- To cite this version:}

Tatiana Manrique-Espindola, Hugo Malaise, Mirko Fiacchini, Thomas Chambrion, Gilles Millérioux. Model predictive real-time controller for a low-consumption electric vehicle. 2nd International Symposium on Environement-Friendly Energies and Applications, EFEA 2012, Jun 2012, Newcastle, United Kingdom. pp.CDROM. hal-00709244

\section{HAL Id: hal-00709244 https://hal.science/hal-00709244}

Submitted on 18 Jun 2012

HAL is a multi-disciplinary open access archive for the deposit and dissemination of scientific research documents, whether they are published or not. The documents may come from teaching and research institutions in France or abroad, or from public or private research centers.
L'archive ouverte pluridisciplinaire $\mathbf{H A L}$, est destinée au dépôt et à la diffusion de documents scientifiques de niveau recherche, publiés ou non, émanant des établissements d'enseignement et de recherche français ou étrangers, des laboratoires publics ou privés. 


\title{
Model Predictive Real-Time Controller for a Low-Consumption Electric Vehicle
}

\author{
Manrique T. ${ }^{1}$, Malaise $\mathrm{H}^{2}{ }^{2}$, Fiacchini M. ${ }^{1}$, Chambrion T. ${ }^{3,2}$, Millerioux G. ${ }^{1}$ \\ ${ }^{1}$ Université de Lorraine, Centre de Recherche en Automatique de Nancy, Vandœuvre, F-54506, France \\ ${ }^{2}$ INRIA, Villers-lès-Nancy, F-54600, France \\ ${ }^{3}$ Université de Lorraine, Institut Elie Cartan de Nancy, Vandœuvre, F-54506, France
}

\begin{abstract}
It is described a complete benchmark designed for testing performances of an electric vehicle in terms of consumption during the European Shell Eco-marathon race. A model of the electric vehicle built for the race is first obtained. Then, a low consumption driving strategy is derived. The tracking performances are tested on the electric Vir'Volt benchmark prototype, wherein a Model Predictive real-time Controller has been implemented. The experimental result are detailed.

Index Terms-Electric vehicle, Model Predictive Control, realtime application
\end{abstract}

\section{INTRODUCTION}

The principle of hybridization is to use several different modes of power supply together or separately according to the power requirements. The main of those requirements is to reduce harmful emissions. Research of energy efficiency in the field of transport has been carried out from few decades in the industry (diesel-electric locomotives) and more recently for the general public market (private hybrids cars are available for sale since 1997) [1] [2]. Many specific engineering issues arise, the more recent are energetic efficiency and real time implementation. Of special interest is the problem of how using the different energy sources so that the energy efficiency can be maximize or, in other words, how the vehicle must be driven so that the minimum quantity of fuel is used, the real time implementation constraints being taken into account [1] [3].

With the aim of promoting the research and innovation around the solution of the low consumption problem, the European Shell Eco-Marathon brings together over 200 teams from high schools and universities from all over Europe, in a race involving ecological and economical vehicles. The principle of the race is to go through a number of kilometres in a limited time and with the lower consumption. Several categories are distinguished according to the energy source: fuel cell (hydrogen), battery, solar energy.

The Ecole Supérieure des Sciences et Technologies de l'Ingénieur de Nancy (ESSTIN), has been involved in the European Shell Eco-marathon race since 12 years in the respective category fuel cell and battery. The performances of the team called EcoMotionTeam have been improved very significantly over the years and now regularly reach places of honour in the contest. In addition, the team was often awarded with special prizes such as the price of technical innovation, the price of the security or the price of National Education.
Motivated by higher ambitious results, researchers from IECN, CRAN and INRIA have joined the EcomotionTeam this year. The aim of this paper is to show the effectiveness, in terms of consumption, of a Model Predictive Control implemented into the vehicle and tested on a benchmark which is intended to reproduce as close as possible the vehicles during the race. The motivation of resorting to a MPC-based controller lies in that they are known to have appealing ability to ensure robustness despite the uncertainties in the model and are amenable to incorporate constraints (saturation of the actuators for example) [1].

This paper is organized as follows: In Sections II and III, the context of the present investigation and the prototype called Vir'Volt are presented. Section IV details the MPC algorithm that will achieve the tracking of the driving strategy. Finally, Section V presents the implementation of the low consumption strategy and the MPC real time controller into the vehicle and benchmark, and the experimental results are provided. Section VI is devoted to a conclusion and sketches future works.

\section{CONTEXT}

The EcoMotionTeam has developped successive vehicles over the past 12 years. The prototype named Vir'Volt is the fifth generation of vehicles developed by the EcoMotionTeam. This recent prototype (see Figure 1) has been ranked last year 2nd in the Plug-in (battery) category among 12 others vehicles and 7 th among the 100 participants of the European competition with a result of $532 \mathrm{~km} / \mathrm{kWh}$ (equivalent to $4732 \mathrm{~km}$ with one litre of fuel). The prototype is a three wheels vehicle, the direction is made by the front wheel and the propulsion by one of the two rear wheels. It can reach the speed of $35 \mathrm{~km} / \mathrm{h}$ and the energy is given by a $24 \mathrm{~V}$ battery to an electric motor which develops $0,4 \mathrm{Nm}$. This vehicle has many electronics systems in order to communicate with the pit stop. The total weight of the car is $40 \mathrm{~kg}$, the pilot needs to weight at least $50 \mathrm{~kg}$ according to the Shell Eco-marathon rules, thus total mass of the vehicle is $90 \mathrm{~kg}$.

So far, the driving strategy proposed by the EcoMotionTeam was a stop-and-go one. It merely reduced to manually turn on or off the engine by the pilot according to whether the vehicle was going up or down respectively. However, the new and ambitious challenges in terms of the consumption strategy requires more complex driving strategies and the 


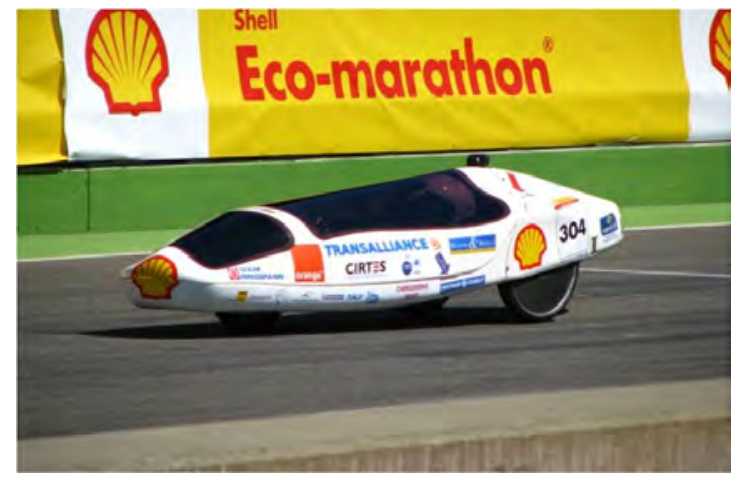

Fig. 1. The Vir'Volt prototype in the Shell Eco-marathon 2011.

accuracy of the tracking is decisive in the final performances.

In 2011, the race was held in Lausitz, Germany. The Lausitz's track could be considered like a straight line since their was no deceleration or complex curves. Then the driving instructions corresponding to the strategy could be follow easily by the pilot. The only strategy was so to maintain a fixed average speed along the track. The efficiency of the converter/motor being better when working at full regime, the strategy consisted in driving around an average value by accelerating at maximum until a maximum speed was reached and then decelerating until the vehicle reached a minimum speed.

On the other hand, in the Rotterdam's 2012 street circuit, the new track require high decelerations due to chicanes and to deal with an expected resulting high traffic. Then, it is clear that an advanced strategy and a control are required in order to gain efficiency.

Achieving a low consumption requires three central works. First, it must be obtained a valuable model of the vehicle. Secondly, deriving a reference driving trajectory in terms of expected position and velocity all along the circuit. Both tasks can be performed off-line. Finally, a powerful tracking strategy must be designed and implemented so that it can works in real-time. The following sections addressed all of these tasks. For the identification of the vehicle and the computation of the reference trajectory, an application called SimStrat (see examples of captured screens of the graphical interface in Figure 2) has been developed by the EcoMotionTeam.

\section{MODELING}

The dynamics of the vehicle can be described in terms of the internal forces acting over the vehicle and given by the force of traction $F_{\text {traction }}[\mathrm{N}]$ due to the engine, and the external forces due to the aerodynamics $F_{\text {aerodynamics }}[\mathrm{N}]$, the rolling resistance (contact wheel-ground) $F_{\text {rolling }}[\mathrm{N}]$, and the slope resistance $F_{\text {slope }}[\mathrm{N}]$. All this forces are related by Newton's law involving the mass $m[\mathrm{~kg}]$ of the vehicle and its

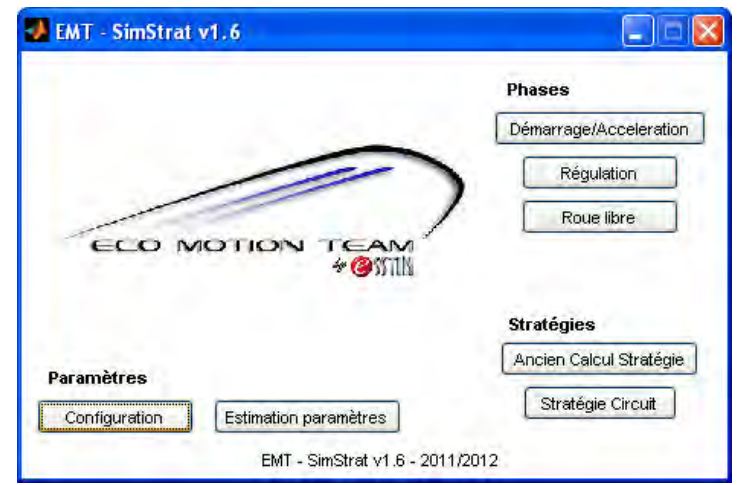

(a) User interface.

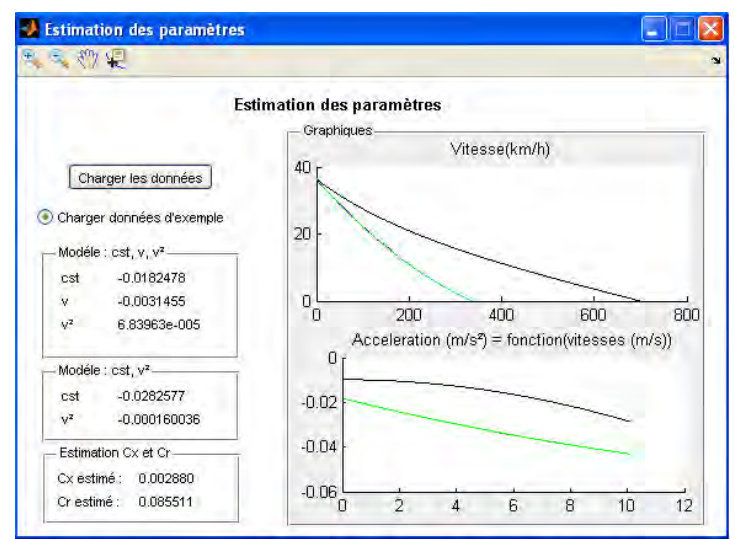

(b) Parameter estimation window.

Fig. 2. Graphical user interface SimStrat designed by the EcoMotionTeam to estimate the vehicle model and find the driving strategy.

acceleration $\frac{d x_{2}(t)}{d t}\left[\mathrm{~m} / \mathrm{s}^{2}\right]$ as $[3]$ :

$$
\begin{aligned}
& m \frac{d x_{2}(t)}{d t}=F_{\text {traction }}-F_{\text {aerodynamics }}-F_{\text {rolling }}-F_{\text {slope }} \\
& m \frac{d x_{2}(t)}{d t}=F_{\text {traction }}-\frac{1}{2} \rho C_{x} S x_{2}(t)^{2}-m g N_{r}-g x_{2}(t) \frac{d \theta}{d x_{1}(t)}
\end{aligned}
$$

with $x_{1}(t)$ the position in $[\mathrm{m}]$ and $x_{2}(t)$ the velocity of the vehicle in $[\mathrm{m} / \mathrm{s}]$. The aerodynamic force $F_{\text {aerodynamics }}$ is given in terms of the frontal area $S\left[\mathrm{~m}^{2}\right]$ of the vehicle, the aerodynamic drag coefficient $C_{x}$, and the air density $\rho\left[\mathrm{kg} / \mathrm{m}^{3}\right]$. The rolling force $F_{\text {rolling }}$ is given in terms of the rolling resistance coefficient $N_{r}$, and the slope resistance force $F_{\text {slope }}$ is given in terms of the variation of the slope $\theta . g\left[\mathrm{~m} / \mathrm{s}^{2}\right]$ is the gravitation acceleration.

The traction force is given by the torque applied by the engine and the resistance of the connecting rod in the crankshaft. This resistance in given by $C_{\text {pivot }} / R$, with $C_{\text {pivot }}[\mathrm{Nm}]$ the bearing resistance and the $R[\mathrm{~m}]$ the radius of the wheel. When the engine is working, the torque due to the motor is $C_{m o t}[\mathrm{Nm}]$, and if it is not working the new torque applied is $C_{\text {min }}[\mathrm{Nm}]$. By introducing the input $u(t) \in[0,1]$ (duty cycle), such as $u(t)$ is the portion of a switching period in which the engine is on, and $(1-u(t))$ the portion of period while the 
engine is off, then the traction force can be written by:

$$
F_{\text {traction }}=\frac{u(t) C_{m o t}+(1-u(t)) C_{m i n}}{R}-\frac{C_{\text {pivot }}}{R}
$$

Considering that the path profile has no elevations (flat path) or that the slope remains always constant, from (1) and (2), the nonlinear dynamics of the vehicle can be written as follows:

$$
\dot{x}_{1}=x_{2}
$$

$\dot{x}_{2}=\frac{u(t) C_{m o t}+(1-u(t)) C_{m i n}-C_{p i v o t}}{m R}-N_{r} g-\frac{1}{2} \frac{\rho C_{x} S}{m} x_{2}{ }^{2}$

\section{A. The linear discrete model}

For practical purposes, a discrete model should be obtained. To this end, the nonlinear model (3)-(4) is discretized by using the Euler's Forward difference numerical approximation that is written in an explicit way, and not in an implicit way like the Tustin's method or Euler's backward discretizing method e.g [4].

Forward differentiation Euler's Method: $\dot{x}\left(t_{k}\right)=\frac{x\left(t_{k+1}\right)-x\left(t_{k}\right)}{T_{s}}$

Backward differentiation Euler's Method: $\dot{x}\left(t_{k}\right)=\frac{x\left(t_{k}\right)-x\left(t_{k-1}\right)}{T_{s}}$

with $T_{s}$ the sample time. The Euler's Forward difference method makes easier the discretizing process of nonlinear functions since gives an explicit or isolated expression for the state variable $x(t)$ in an direct way [4].

From (3)-(4) and (5), the discrete state space model is obtained for the position and the velocity, considered respectively as the first and the second component of the state vector and denoted $x_{1}(k)$ and $x_{2}(k)$, by using the Euler's Forward method as:

$$
\begin{gathered}
x_{1}(k)=x_{1}(k-1)+T_{s} x_{2}(k-1) \\
x_{2}(k)=x_{2}(k-1)+T_{s} \frac{\left(C_{m o t}-C_{m i n}\right) u(k-1)+C_{m i n}-C_{\text {pivot }}}{m R} \\
-T_{s} N_{r} g-T_{s} \frac{1}{2} \frac{\rho C_{x} S}{m} x_{2}(k-1)^{2}
\end{gathered}
$$

When the discretizing process is implemented, a zero holder is usually considered and introduces a delay $T_{s} / 2$. This delay can influence the stability of the system if it is too large. In [4], it is demonstrated that the effect of the time delay over the system stability is tolerable if the time delay $T_{s} / 2$ is smaller than one tenth of the rise time $t_{r}$ ( $63 \%$ rise time rule) of the system, i.e:

$$
T_{s} \leq \frac{t_{r}}{5}
$$

As can be seen in (7), a nonlinear differential equation produces a nonlinear difference equations. By taking $u_{e} \in$ $c o(\{0,1\})$ as the duty cycle of the averaged input that takes (7) to the equilibrium point $x_{2 e}$ :

$$
x_{2 e}=\sqrt{2 \cdot \frac{u_{e}-\left(C_{\text {pivot }}+N_{r} g m R\right)}{\rho C_{x} S R}}
$$

Then the linearized discrete-time state space dynamics is given by:

$$
\hat{x}(k)=\mathbf{A} \hat{x}(k-1)+\mathbf{B} \hat{u}(k-1)
$$

where $\hat{x}(k)=\left[x_{1}(k), x_{2}(k)-x_{2 e}\right]^{T} \in \mathbb{R}^{n}$ (with $n=2$ ), $\hat{u}(k)=u(k)-u_{e}$ and:

$$
\mathbf{A}=\left[\begin{array}{cc}
1 & T s \\
0 & 1-\frac{2 b T s}{m} x_{2 e}
\end{array}\right] \quad \mathbf{B}=\left[\begin{array}{c}
0 \\
\frac{\left(C_{m o t}-C_{m i n}\right)}{m R} T s
\end{array}\right]
$$

The output equation is the linearized velocity $\hat{x}_{2}(k)=$ $x_{2}(k)-x_{2 e}$ and reads

$$
y(k)=\mathbf{C} \hat{x}(k-1), \text { where } \mathbf{C}=\left[\begin{array}{ll}
0 & 1
\end{array}\right]
$$

In Figure 3 are plotted the velocity $x_{2 e}$ with respect to the controls $u_{e}$ in the admissible range $[0,1]$ and corresponds to the nonlinear characteristic (9). An example of linearization around a control operating point is also depicted.

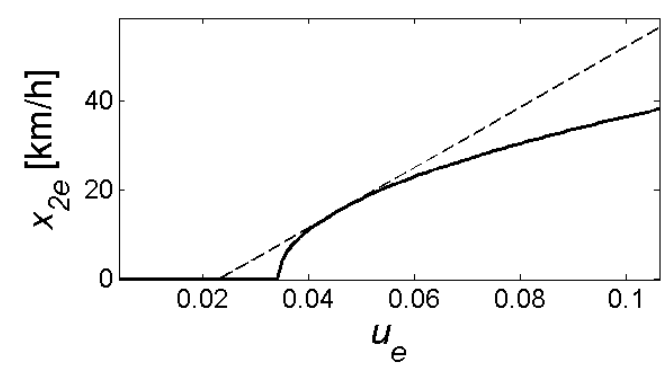

Fig. 3. Velocity $x_{2 e}$ with respect to control $u_{e}$ for the nonlinear model (continuous line) and the linearized model (dashed line)

\section{Model Predictive Control}

\section{A. Driving Strategy}

For a prescribed circuit, the driving strategy is a collection of finite numbers of pairs $\left(x_{1}^{*}(k), x_{2}^{*}(k)\right)$ where $x_{2}^{*}(k)$ corresponds to the required velocity assigned to the position $x_{1}^{*}(k)$ in the circuit at time $k$, so as a minimal consumption is achieved. We define the driving strategy state vector as

$$
x^{*}(k)=\left[x_{1}^{*}(k), x_{2}^{*}(k)\right]^{T}
$$

The search for the driving strategy is an iterative procedure which, given the model of the vehicle, the road profile (slope, curves, ...) and the constraints in terms of maximum velocity allowed at each curve, maximum time of the race, total number of kilometers, ..., must return (12) for every $k$. Then, for each $x_{2}^{*}(k)$, the corresponding required control $u^{*}(k)$ which guarantees that the actual velocity $x_{2}(k)$ is as close as possible of $x_{2}^{*}(k)$ (tracking) can be derived by solving (9) with $x_{2 e}=$ $x_{2}^{*}(k)$ and $u_{e}=u^{*}(k)$, at each $k$. An example of driving strategy is depicted in the captured screen of Figure 4. 


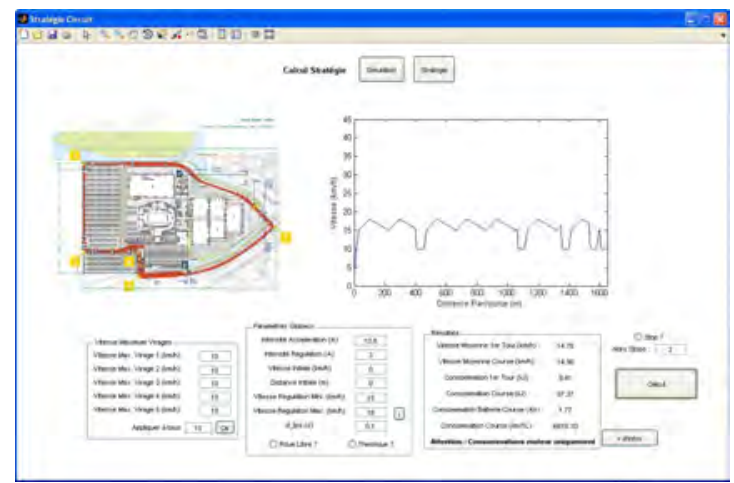

Fig. 4. Rotterdam's 2012 street circuit (Courtesy of Shell) and a corresponding low consumption driving strategy.

\section{B. Tracking}

This subsection addresses the problem of driving the vehicle in real-time in order to guarantee that the state $x(k)=$ $\left[x_{1}(k), x_{2}(k)\right]^{T} \in \mathbb{R}^{2}$ of the vehicle remains as close as possible to the driving strategy $x^{*}(12)$. As pointed out in the introduction, a Model Predictive Control approach is well suited for that purpose.

Let us define $\Delta \tilde{u}(k)=u(k)-u^{*}(k)$ as the distance between the predicted input $u(k)$ and the control solution $u^{*}(k)$ at the instant $k$. Since at every sampling instant $k$, the state $x(k)$ can be measured, then for a prediction horizon of length $N_{p}$, the vector $\Delta \mathbf{U} \in \mathbb{R}^{N_{p} \times 1}$ which should achieve the tracking can be defined as:

$$
\Delta \mathbf{U}=\left[\Delta \tilde{u}(k), \Delta \tilde{u}(k+1), \Delta \tilde{u}(k+2), \ldots, \Delta \tilde{u}\left(k+N_{p}-1\right)\right]^{T}
$$

Furthermore, let us define $\Delta \tilde{x}(k)=x(k)-x^{*}(k)$ as the distance between the predicted state $x(k)$ and the state solution $x^{*}(k)$ at the instant $k$. The predicted state variables are then given by:

$\Delta \mathbf{X}=\left[\Delta \tilde{x}(k+1)^{T}, \Delta \tilde{x}(k+2)^{T}, \Delta \tilde{x}(k+3)^{T}, \ldots, \Delta \tilde{x}\left(k+N_{p}\right)^{T}\right]^{T}$

The relationship between $\Delta \mathbf{X} \in \mathbb{R}^{n N_{p} \times 1}$ and $\Delta \mathbf{U}$ is given by:

$$
\Delta \mathbf{X}=\mathbf{F}\left(x(k)-x^{*}(k)\right)+\Phi \Delta \mathbf{U}
$$

with $\mathbf{F} \in \mathbb{R}^{n N_{p} \times n}$ and $\Phi \in \mathbb{R}^{n N_{p} \times N_{p}}$ (with $n=2$ ) where:

$\mathbf{F}=\left[\begin{array}{c}\mathbf{A} \\ \mathbf{A}^{2} \\ \mathbf{A}^{3} \\ \vdots \\ \mathbf{A}^{N_{p}}\end{array}\right] \quad \Phi=\left[\begin{array}{ccccc}\mathbf{B} & 0 & 0 & \ldots & 0 \\ \mathbf{A B} & \mathbf{B} & 0 & \ldots & 0 \\ \mathbf{A}^{2} \mathbf{B} & \mathbf{A B} & \mathbf{B} & \ldots & 0 \\ \vdots & \vdots & \vdots & \ddots & \vdots \\ \mathbf{A}^{N_{p}-1} \mathbf{B} & \mathbf{A}^{N_{p}-2} \mathbf{B} & \mathbf{A}^{N_{p}-3} \mathbf{B} & \ldots & \mathbf{B}\end{array}\right]$

The MPC-based controller, which is intended to be embedded on-board during the race, must guarantee the tracking of the driving strategy despite unpredictable events like emergency brakes, wind, .... The control must be the solution of the following quadratic criterion:

$$
\begin{aligned}
\min _{\Delta \mathbf{U}}\left(\mathbf{J}=\Delta \mathbf{X}^{T} Q \Delta \mathbf{X}+\Delta \mathbf{U}^{T} P \Delta \mathbf{U}\right) \\
\text { s.t. } \Delta \mathbf{X}=\mathbf{F}\left(x(k)-x^{*}(k)\right)+\Phi \Delta \mathbf{U} \\
x(0)=x_{\text {ini }} \quad u \in[0,1]
\end{aligned}
$$

with $P \in \mathbb{R}^{N_{p} \times N_{p}}$ and $Q \in \mathbb{R}^{n N_{p} \times n N_{p}}$ diagonal matrices.

Plugging (15) into (17), it can be shown after some basic manipulations that the minimization problem (17) can be reformulated in a strictly equivalent way and reads:

$$
\begin{aligned}
\min _{\Delta \mathbf{U}} \frac{1}{2} \Delta \mathbf{U}^{T} \mathbf{H} \Delta \mathbf{U}+\mathbf{f}^{T} \Delta \mathbf{U} \\
\text { s.t. } M \Delta \mathbf{U} \leq N
\end{aligned}
$$

where $\mathbf{H} \in \mathbb{R}^{N_{p} \times N_{p}}$ and $\mathbf{f} \in \mathbb{R}^{N_{p} \times 1}$ are given by:

$$
H=\Phi^{T} Q \Phi+P \text { and } \mathbf{f}=\Phi^{T} Q \mathbf{F}\left(x(k)-x_{k}^{*}\right)
$$

The constraint $M \Delta \mathbf{U} \leq N$ in (18) must be written in terms of the constraints required for the state (related to the reference tracking error) and the constraints required for the input (related to requirement $u(k) \in[0,1]$ ). Firstly, the constrains of the state are expressed as:

$$
M_{x} \Delta \mathbf{X} \leq N_{x}
$$

where $M_{x} \in \mathbb{R}^{2 n N_{p} \times n N_{p}}$ and $N_{x} \in \mathbb{R}^{2 n N_{p} \times 1}$ are given by:

$$
M_{x}=\left[\begin{array}{cccc}
\mathbf{I}_{n \times n} & 0 & \ldots & 0 \\
0 & \mathbf{I}_{n \times n} & \ldots & 0 \\
\vdots & \vdots & \ddots & \vdots \\
0 & 0 & \ldots & \mathbf{I}_{n \times n} \\
-\mathbf{I}_{n \times n} & 0 & \ldots & 0 \\
0 & -\mathbf{I}_{n \times n} & \ldots & 0 \\
\vdots & \vdots & \ddots & \vdots \\
0 & 0 & \ldots & -\mathbf{I}_{n \times n}
\end{array}\right] \quad N_{x}=\left[\begin{array}{c}
\max _{\Delta x} \\
\max _{\Delta x} \\
\vdots \\
\max _{\Delta x} \\
\min _{\Delta x} \\
\min _{\Delta x} \\
\vdots \\
\min _{\Delta x}
\end{array}\right]
$$

with $\mathbf{I}_{n \times n} \in \mathbb{R}^{n \times n}$ the identity matrix, and $\max _{\Delta x} \in \mathbb{R}^{n \times 1}$ and $\min _{\Delta x} \in \mathbb{R}^{n \times 1}$ the upper and lower limits desired for the tracking error $\Delta \tilde{x}(k)$, such as:

$$
\min _{\Delta x} \leq \Delta \tilde{x}(k)=x(k)-x^{*}(k) \leq \max _{\Delta x}
$$

By using (15), the inequality in (20) can be rewritten in terms of $\Delta \mathbf{U}$, as:

$$
M_{x} \Phi \Delta \mathbf{U} \leq N_{x}-M_{x} \mathbf{F} \Delta \tilde{x}(k)
$$

On the other hand, the constrains for the input can be written as:

$$
M_{u} \Delta \mathbf{U} \leq N_{u}
$$

where $M_{u} \in \mathbb{R}^{2 N_{p} \times N_{p}}$ and $N_{u} \in \mathbb{R}^{2 N_{p} \times 1}$ are given by:

$$
M_{u}=\left[\begin{array}{c}
\mathbf{I}_{N_{p} \times N_{p}} \\
-\mathbf{I}_{N_{p} \times N_{p}}
\end{array}\right] \quad N_{u}=\left[\begin{array}{c}
\max _{\Delta u} \\
\vdots \\
\max _{\Delta u} \\
\min _{\Delta u} \\
\vdots \\
\min _{\Delta u}
\end{array}\right]
$$

with $\mathbf{I}_{N_{p} \times N_{p}} \in \mathbb{R}^{N_{p} \times N_{p}}$ the identity matrix, and $\max _{\Delta u} \in$ $\mathbb{R}^{1 \times 1}$ and $\min _{\Delta u} \in \mathbb{R}^{1 \times 1}$ such as:

$$
\min _{\Delta u} \leq \Delta \tilde{u}(k) \leq \max _{\Delta u}
$$

The values $\max _{\Delta u}$ and $\min _{\Delta u}$ must be expressed such as the condition $u(k) \in[0,1]$ is always satisfied. By making:

$$
\min _{\Delta u}=0-u^{*}(k) \text { and } \max _{\Delta u}=1-u^{*}(k)
$$


then, from (26) it is obtained:

$$
0 \leq u(k) \leq 1
$$

Finally from (23) and (24), $M$ and $N$ in (18) are expressed as:

$$
M=\left[\begin{array}{c}
M_{x} \Phi \\
M_{u}
\end{array}\right] \quad N=\left[\begin{array}{c}
N_{x}-M_{x} \mathbf{F} \Delta \tilde{x}(k) \\
N_{u}
\end{array}\right]
$$

\section{EXPERIMENTAL RESULTS}

\section{A. Parameter estimation}

The nonlinear model (1) involves two unknown parameters $C_{x}$ and $N_{r}$. For the estimation of those parameters, multiple decelerations to zero have been performed for the Vir'Volt vehicle. It has been done by turning off the motor $\left(F_{\text {traction }}=0\right)$ after accelerating the vehicle until a maximum velocity $V e l_{\text {ini }}$ in a low slope variations road and without curves.

For the Vir'Volt vehicle and its environment, the physical known parameters are the gravity acceleration $g=9.81\left[\mathrm{~m} / \mathrm{s}^{2}\right]$, the wheel radius $R=0.24 \mathrm{~m}$, the frontal surface $S=$ $0.275 \mathrm{~m}^{2}$, the total mass $m=90 \mathrm{~kg}$, the air density $\rho=$ $1.225\left[\mathrm{~kg} / \mathrm{m}^{3}\right], C_{\text {pivot }}$ being neglected. The initial velocity is $V e l_{\text {ini }}=40 \mathrm{~km} / \mathrm{h}$. The data bank and the corresponding best fitting in terms of Mean Square Error (MSE) regarding (1) is depicted in Figure 5.

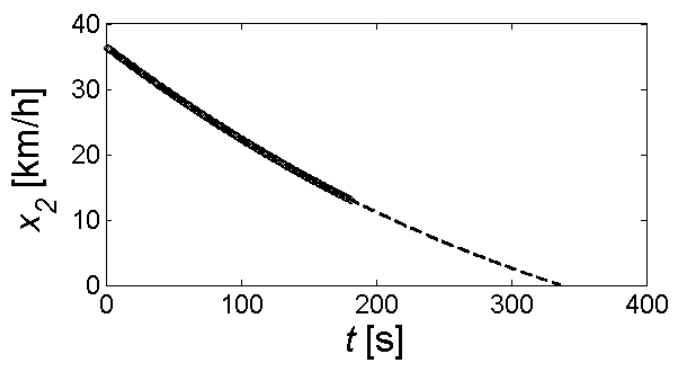

Fig. 5. Decelerating data bank in circles and best fitting in dashed line for the Vir'Volt vehicle.

The experiments gives $C_{x}=0.085$ and $N_{r}=0.0029$. In Figure 6 is depicted the simulated step response of the resulting nonlinear model (4) for a step input of $u=0.045$. The $63 \%$ response time of the nonlinear system in $t_{r}=935.5 \mathrm{~s}$. From now on $C_{m o t}=6.228[\mathrm{Nm}]$ and $C_{m i n}=0[\mathrm{Nm}]$.

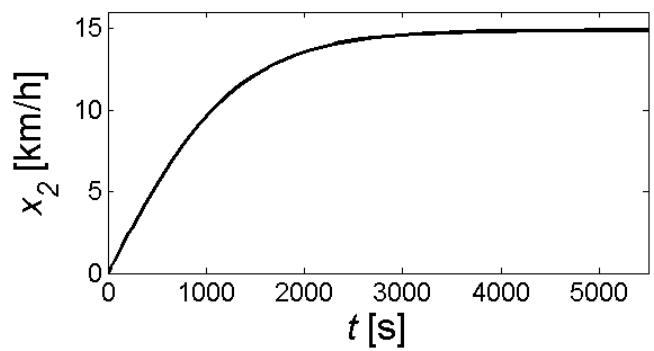

Fig. 6. Nonlinear system step response.
Finally, the linear dicrete model (10) has been obtained by considering a sampled time $T_{s}=0.2 \mathrm{~s}$ which fulfills (8), and the operating point $u_{e}=0.040$ and $x_{2 e}=11 \mathrm{~km} / \mathrm{h}$ in (9).

\section{B. Benchmark}

In order to assess the efficiency of the control law, a benchmark emulating both the vehicle and the circuit profile has been built (see Figure 7). The benchmark is composed

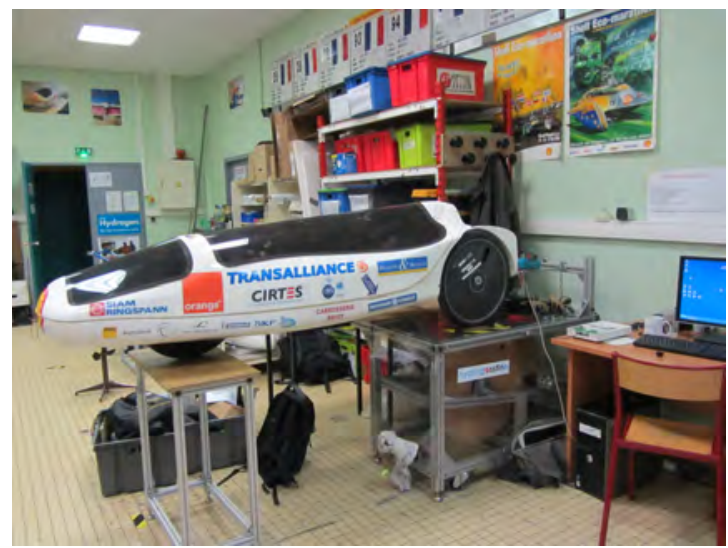

(a) Vir'Volt vehicle, benchmark and DAQ system.

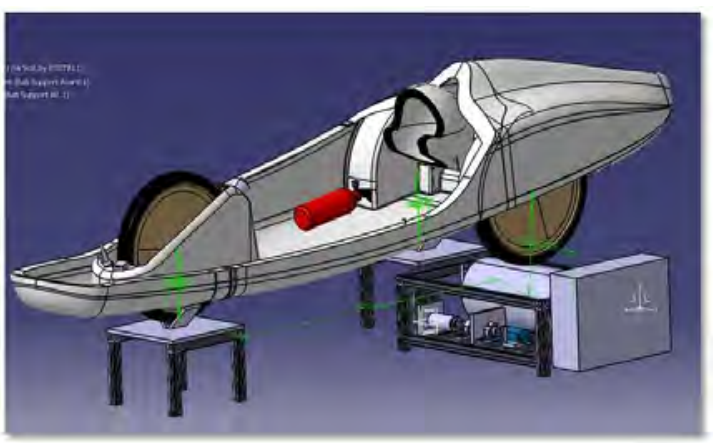

(b) CAD benchmark.

Fig. 7. Benchmark

of two inertia wheels over which the vehicle is placed. The inertia wheels allows for emulating the intertia of the vehicle. This benchmark has an electric brake and a motor in order to simulate slopes, wind and others disturbances such as the friction torque in the wheels. The benchmark is also equipped by a a torque-meter. The energy consumed by the vehicle is measured by plugging an energy-meter onto the battery.

The data acquisition system (DAQ) used to probe the velocity and the current position of the vehicle consists in a National Instruments analog DAQ system (USB-6289) which receives data from the vehicle (such as velocity, position, current, voltage, ...). The MPC computation, in particular the quadratic solver corresponding to (19) is developed by a python script based on the mathematics $\mathrm{C}$ library CVXOPT, BLAS and LAPACK. The MPC is run on a Linux-embedded computer named APF51 developed by the company Armadeus. This embedded computer is based on an ARMv7 (working frequency $800 \mathrm{Mhz}$ ) processor and will, after computation, 
send a command to the motor according to the velocity and position of the car.

\section{Tracking experimental results}

The Rotterdam 2012 circuit has a total length of $16.3 \mathrm{~km}$ and five $90^{\circ}$ curves with lengths between $19 \mathrm{~m}$ and $44 \mathrm{~m}$. The resulting driving strategy obtained by means of the aforementioned application SimStrat is depicted in Figure 8a. The constraints were: maximum motor current value $8 \mathrm{~A}$ (and thus $C m o t$ ), maximum velocity regulation $13 \mathrm{~km} / \mathrm{h}$, minimum velocity regulation $10 \mathrm{~km} / \mathrm{h}$ and velocity in curves $8 \mathrm{~km} / \mathrm{h}$. The estimated consumption for this driving strategy is $9.2 \mathrm{~kJ}$ (equivalent to $640 \mathrm{~km} / \mathrm{kWh}$ or $6278 \mathrm{~km} / 1 \mathrm{Lfuel}$ ) and the average velocity is $11 \mathrm{~km} / \mathrm{h}$.

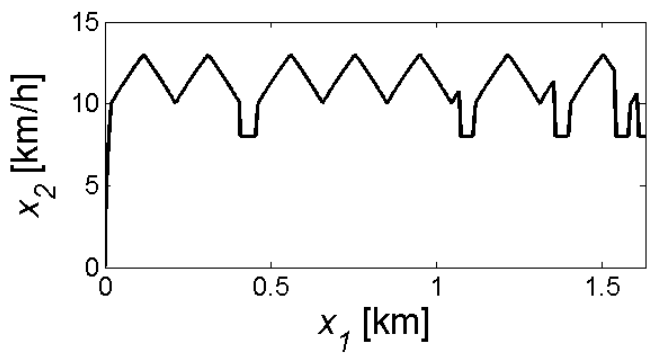

(a) Driving strategy.

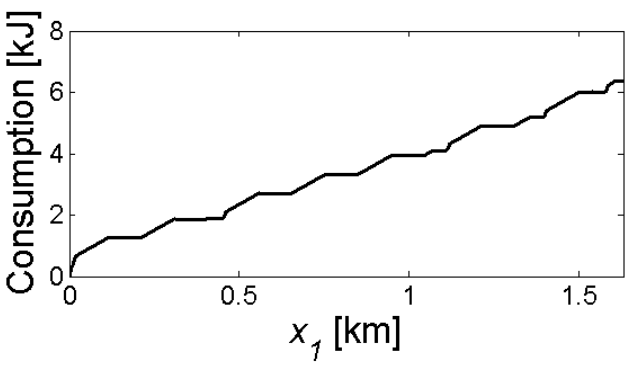

(b) Estimated energy consumption.

Fig. 8. Low consumption driving strategy with total consumption $9.2 \mathrm{~kJ}$. $x_{2}[\mathrm{~km} / \mathrm{h}]$ is the velocity and $x_{1}[\mathrm{~km}]$ is the position.

The MPC real-time controller has been tested firstly for the tracking of a non-variable reference and secondly for the tracking of the low consumption strategy found in Figure 8, with the MPC state restrictions set as $\min _{\Delta x}=-5 \mathrm{~km} / \mathrm{h}$ and $\max _{\Delta x}=5 \mathrm{~km} / \mathrm{h}$ (see (22)). The matrices $Q$ and $P$ in (17) are unitary matrices.

Results shown in Figure 9 are achieved for the velocity and the control signal for the tracking of a reference of $x_{2}$ ref $=15 \mathrm{~km} / \mathrm{h}$ from initial condition $x_{2}$ ini $=0 \mathrm{~km} / \mathrm{h}$. The total consumption was $3,5676 \mathrm{~kJ}$. As it can be observed the control signal in Figure $9 \mathrm{~b}$ accomplishes the control constraints imposed by (28). The stable state error is $0.44 \mathrm{~km} / \mathrm{h}$, that corresponds to $2.93 \%$. The time response of the controller is $7 \mathrm{~s}$.

The results shown in Figure 10 point out a good tracking of the driving strategy. The measured energy consumption was $12.1 \mathrm{~kJ}$ (equivalent to $480 \mathrm{~km} / \mathrm{kWh}$ or $4780 \mathrm{~km} / 1 \mathrm{Lfuel}$ ). The difference in the total consumption is due to the fact that

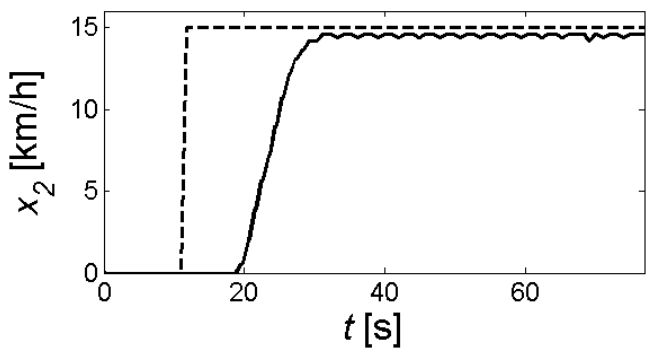

(a) Measured velocity $x_{2}$. In dashed line is the reference.

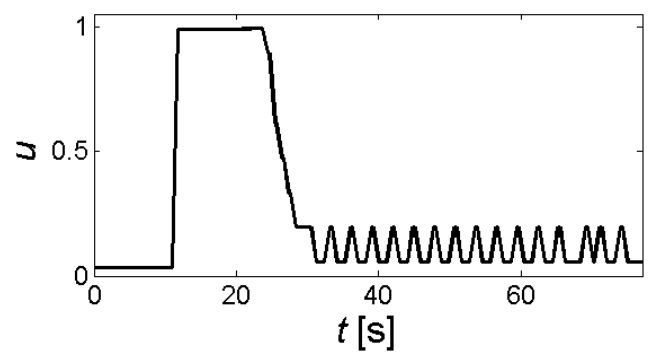

(b) Measured control $u$.

Fig. 9. Tracking experiment for a nonvariable reference.

the efficiency of the engine is not considered here and to the transients before exact tracking.

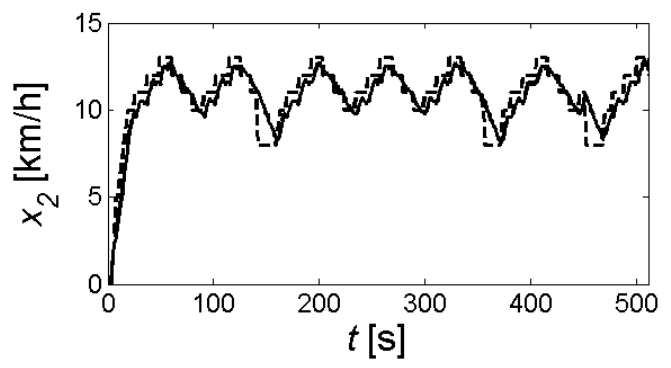

(a) Measured velocity $x_{2}$. In dashed line is the reference.

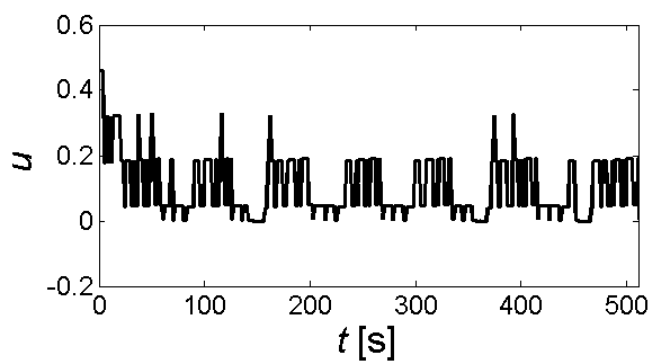

(b) Measured control $u$.

Fig. 10. Tracking experiment for the driving strategy.

\section{CONCLUSIONS AND FUTURE WORK}

A benchmark for testing a low consumption driving strategy of a vehicle with battery source energy supply has been built. The benchmark is able to emulate the race conditions. A model of the vehicle has been obtained and used to design a Model Predictive Control tracking strategy. The controller has 
been implemented in the vehicle and achieves good tracking performances. As future work, the controller will be tested on board for the actual race.

\section{ACKNOWLEDGMENTS}

It is a pleasure for the authors to thank the students and the teaching staff of EcoMotionTeam and ESSTIN for their valuable help.

The authors would also like to thank the company Armadeus for their sponsorship and help developing embedded solutions.

This work has been supported by CPER Région Lorraine (AOC project), Fédération Charles Hermite, ESSTIN and INRIA.

Third author partially supported by ANR GCM, contract number NT-504590 and ERC StG number 239748.

\section{REFERENCES}

[1] A. Sciarretta and L. Guzzella, "Control of hybrid electric vehicles," IEEE Control Systems Magazine, vol. 27, no. 2, pp. 60-70, 2007.

[2] C. Bordons, M. A. Ridao, A. Pérez, A. Arce, and D. Marcos, "Model Predictive Control for power management in hybrid fuel cell vehicles," in Vehicle Power and Propulsion IEEE Conference, 2010.

[3] J. Santin, The world's most fuel efficient vehicle: design and development of PAC-Car II. vdf Hochschulverlag AG an der ETH Zürich, 2007.

[4] F. Haugen, Advanced Dynamics and Control. TechTeach, 2010. 